\title{
Feasibility Study on Blood Pressure Estimations from Voice Spectrum Analysis
}

\author{
Motoki Sakai \\ School of Information \\ Environment \\ Tokyo Denki University
}

\begin{abstract}
Recently, preventive healthcare has attracted increased attention. Because of a demonstrable mortality rate, hypertension is a disease that should be prevented before it becomes severe. To decrease the mortality rate for hypertension, it is desirable for people to measure their own blood pressure (BP) on a routine basis. At present, many ambulatory BP gauges are available. Yet, it is unlikely that people will carry around an ambulatory BP gauge to measure their BP, even among those with a high risk of illness. Such methods are therefore ineffective for preventive healthcare. Therefore, it would be preferable to measure information related to BP easily and without a dedicated gauge. In this research, a BP estimation method is proposed using voicespectrum analysis. If BP estimations from the voice spectrum are accurate, we may be able to measure BP with a smartphone's voice recorder. To evaluate the feasibility of such BP estimations, two subjects' BPs and voice data were measured, and the correlation coefficients were examined. Results showed that both diastolic and systolic BPs and the spectral component of the voice were not non-correlated ( > $|0.6|)$ at specific frequency bands. To estimate BP levels, a support-vector machine was proposed, and the correlation coefficients between measured and estimated BPs exceeded 0.9. However, individual differences in the voice spectrum were not adequately addressed. In future research, individual differences will be investigated in a study involving more subjects.
\end{abstract}

\section{General Terms}

Data mining, Biomedical engineering

\section{Keywords}

Preventive health, Blood pressure, Voice spectrum, Estimation, Support vector machine

\section{INTRODUCTION}

In recent years, there has been an emphasis on preventive healthcare using measurements taken in a premorbid state. Hypertension is one disease that should be prevented before it becomes severe, because of the associated risk of a cerebral stroke. Some researchers have reported a mortality rate associated with hypertension $[1,2]$, and there are guidelines available for its prevention [3, 4].

One of basic ways to prevent hypertension is to measure blood pressure (BP) on a daily basis. It is also important to be consistently aware of one's own condition, and to take the appropriate measures to reduce the risk of disease. At present, there are several ambulatory BP gauges available for measuring BP. However, it is unnecessary, even foolish, for healthy people to carry around an ambulatory $\mathrm{BP}$ gauge to measure their BP. On the other hand, such measurements are pertinent to the prevention of hypertension, because many healthy people are unaware of their BP until being diagnosed with hypertension. For this reason, it is desirable in terms of preventive healthcare, for information related to BP to be measured easily and without a particular gauge, such as an ambulatory BP measuring instrument. Therefore, a BP estimation method that does not require a dedicated BP gauge is proposed in this research.

In this paper, voice is adopted as the biological information for estimating BP. Voice is often coupled with BP as biological information measured in psychological experiments. For example, research [5,6] suggests that both $\mathrm{BP}$ and voice are associated with stress. In [5], researchers reported that chronic exposure to stress increases BP, and [6] demonstrated that a stress detector can be developed based on the relation between emotion and variations in speech. It follows that BP and voice are related. Moreover, both are often used as an index to evaluate the same phenomena, such as emotion. Thus, the relationship between BP and the voice spectrum must first be examined. Then, the possibilities for estimating BP using the voice spectrum can be discussed. If BP estimation from the voice spectrum is feasible, it will be considerably beneficial in terms of preventive healthcare. Given the accuracy of such a method, BP can be measured with a smartphone's voice recorder, for instance.

\section{MEASUREMENTS OF BP AND VOICE DATA}

To examine the feasibility of BP estimation from voice data, experiments with BP and voice were conducted. Two subjects were studied: a 33-year-old male, and a 25-year-old female.

First, voice was recorded with a voice recorder: an ICD-TX50 produced by SONY. The voice recorder was kept $5 \mathrm{~cm}$ from the subject's mouth, and the subject was asked to sustain the sound [ä] for $7 \mathrm{~s}$. The sampling rate of the voice recording was $44.1 \mathrm{kHz}$, with 16-bit resolution. Immediately following the recording, the subject's diastolic and systolic BP was measured with a BP gauge: a HEM-1010 produced by OMURON. In this manner, the test was repeated 60 times for each subject. During the estimation step, the diastolic and systolic BP is estimated from the recorded voice data.

\section{CORRELATION BETWEEN BP AND THE VOICE SPECTRUM}

To evaluate the relationship between $\mathrm{BP}$ and the voice spectrum, the recorded voice signals were processed with a fast Fourier transform (FFT), and the correlation coefficients between each spectral component of voice ranging from 120 $\mathrm{Hz}$ to $10 \mathrm{kHz}$ and both diastolic and systolic BP measurements were computed using measured 60 datasets.

Figs. 1 and 2 show the correlation coefficient between the systolic BP and the voice spectrum for the male and female 
subjects. Likewise, Figs. 3 and 4 show the correlation coefficient between the diastolic BP and the voice spectrum.

As shown in Fig. 1, the highest correlation coefficient between the systolic BP and the voice spectrum reaches close to 0.6 around $7,000 \mathrm{~Hz}$ (for the male subject). On the other hand, Fig. 2 shows that its correlation coefficient is approximately -0.6 around $6,000 \mathrm{~Hz}$ (for the female subject). These results indicate that the systolic BP and the voice spectrum are not non-correlated. At other frequency bands, decorrelation between the systolic BP and the spectral component of voice was rejected. Similarly, Figs. 3 and 4 illustrate that diastolic BP and the voice spectrum are not noncorrelated at several frequency bands.

These results suggest that systolic and diastolic BP can be estimated from the voice spectrum, but these correlations will not suffice for such estimations using a weak regression algorithm such as linear regression. In Section 4, an estimation method based on support vector machines (SVMs) is thus proposed.

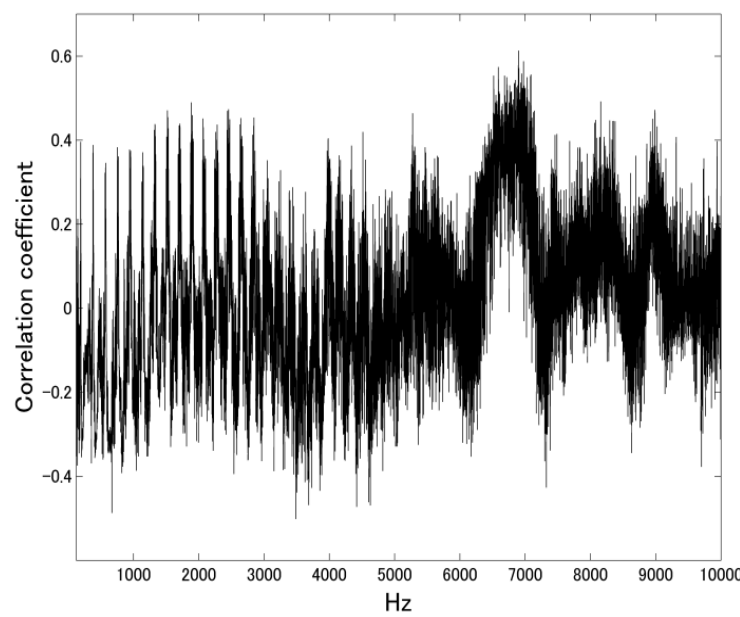

Fig 1: Correlation coefficient between the male subject's systolic BP and his voice

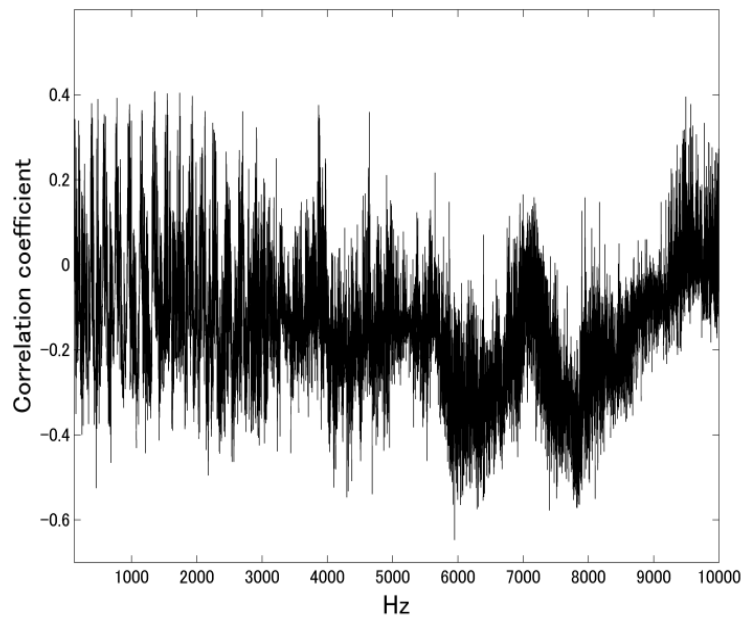

Fig 2: Correlation coefficient between the female subject's systolic BP and her voice

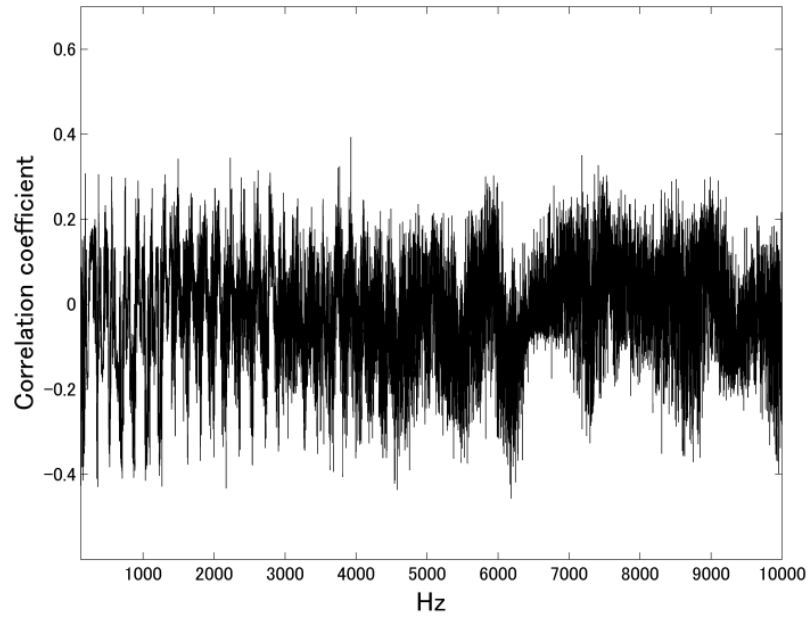

Fig 3: Correlation coefficient between the male subject's diastolic BP and his voice

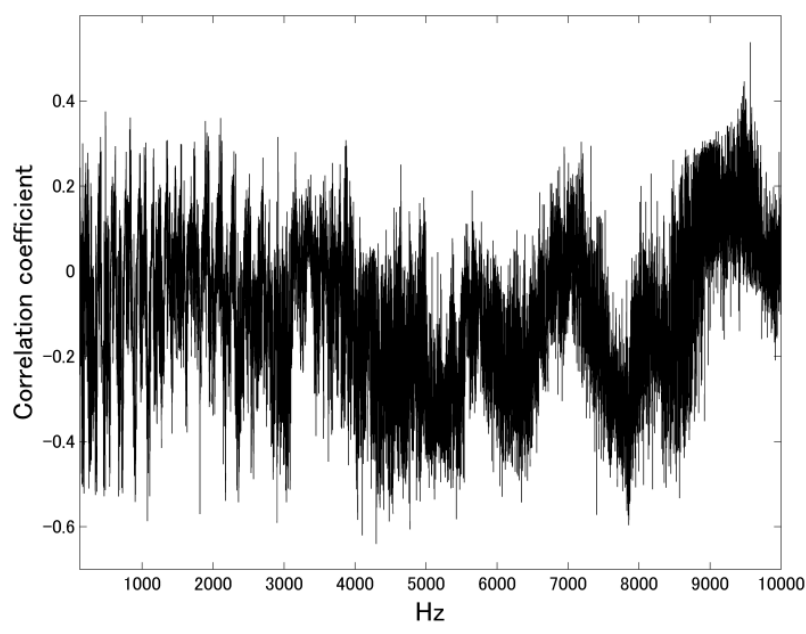

Fig 4: Correlation coefficient between the female subject's diastolic BP and her voice

\section{KERNEL RIDGE REGRESSION- BASED BP ESTIMATION METHOD}

A processing flow for BP estimations from voice-spectrum analysis is shown in Fig. 5. First, the recorded voice signal is transformed into frequency representations with the FFT. Next, the effective spectral components from the voice recordings are selected for $\mathrm{BP}$ estimations by sequential feature selection (SFS) [7]. These spectral components are used as explanatory variables in the subsequent estimation step. Finally, an SVM-based [8, 9] BP estimation is performed using the explanatory variables to the spectral components of the voice. These algorithms will be described in Sections 4.1 and 4.2. 


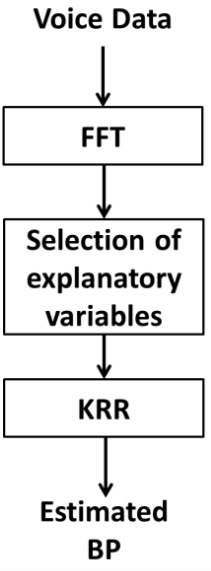

Fig 5: Processing flow for BP estimation

\subsection{Selecting the Explanatory Variables}

The method for selecting suitable explanatory variables within the voice spectrum is described here. An "explanatory variable" is also known as a "feature," but the latter term is reserved for SVM-based explanations to avoid confusion. In this paper, the input variables for the SVM are termed the "explanatory variables."

In most cases, selecting explanatory variables is done for two reasons. The first is to avoid the so-called curse of dimensionality by reducing the complexity of the classification or estimation process. The other is to select the best subset to increase the accuracy of the classification or regression.

In this research, the SFS method was used to increase the accuracy of estimations. SFS is performed according to the following steps.

I. SFS begins with an evaluation of the accuracy of the estimation for each explanatory variable (i.e., for each spectral component in the voice), identifying the most accurate variable.

II. Then, SFS is performed using a pair of explanatory variables. The most accurate variable identified in the previous step is coupled with each of the other variables from the remaining explanatory variable subset, selecting the pair with the highest degree of accuracy.

III. SFS next evaluates three variables: the pair from Step II and another selected from the remaining subset.

IV. Steps I-III are repeated with four or more explanatory variables.

Generally, the estimation accuracy peaks when the number of selected explanatory variables reaches a certain number. Then the best explanatory-variable subset can be singled out for maximum accuracy in the SFS procedure. Note that the "accuracy" is determined by the correlation coefficient between the estimated BP and the measured BP.

In this research, the spectral components of the voice that are applied to the SFS method are confined to a specific frequency range - the range with a higher correlation coefficient between the BP and spectral component of the voice. For example, the frequency band around $7,000 \mathrm{~Hz}$ was applied to the SFS method to estimate the systolic BP for the male subject.

\subsection{Kernel Ridge Regression}

An SVM is a linear classifier or regression in the feature space mapped by nonlinear transformation, a model proposed by Dr. Vapnik [9]. With an SVM, a kernel function realizes an implicit mapping of the input data into high-dimensional feature space. This is defined as a dot product in the feature space and does not require an understanding of the type of features that are being used. Within the class of SVM-based algorithm, Kernel Ridge Regression (KRR), was proposed to estimate $\mathrm{BP}$. KRR is a ridge-regression method based on some kernel matrices, and it can also be derived from a modified least-squares method [10].

Supposing that $\left(x_{1}, y_{1}\right), \cdots,\left(x_{T}, y_{T}\right)$ is a training set, where $T$ is the number of examples, $x_{t} \in \mathbb{R}^{n}$ are $\mathrm{n}$ dimensional vectors, $y_{t} \in \mathbb{R}$, and $t=1, \ldots, T$. A general linear function is defined as $y=w \cdot x$, where $w \in \mathbb{R}$. With the least-squares method, $w=w_{0}$ is solved, which minimizes the objective function (Eq. 1)

$L_{T}(w)=\sum_{t=1}^{T}\left(y_{t}-w \cdot x_{t}\right)^{2}$

When a new sample $x$ is inputted, the predicted label is computed as $w_{0} \cdot x$. Ridge regression, then, replaces Eq. 1 with Eq. 2.

$\lambda\|\boldsymbol{w}\|^{2}+\sum_{t=1}^{T}\left(y_{t}-\boldsymbol{w} \cdot \boldsymbol{x}_{t}\right)^{2}$

where $\lambda$ is a fixed positive constant. This optimization problem can be re-expressed as follows:

$\lambda\|\boldsymbol{w}\|^{2}+\sum_{t=1}^{T} \xi_{t}^{2}$

subject to

$y_{t}-\boldsymbol{w} \cdot \boldsymbol{x}_{t}=\xi_{t}, t=1, \ldots, T$.

To solve Eq. 3 under the constraints in Eq. 4, the Lagrangian equation (Eq. 5) is introduced.

$L(\boldsymbol{w}, \boldsymbol{\xi}, \boldsymbol{\alpha})=\lambda\|\boldsymbol{w}\|^{2}+\sum_{t=1}^{T} \xi_{t}^{2}+\sum_{t=1}^{T} \alpha_{t}\left(y_{t}-\right.$ $\left.\boldsymbol{w} \cdot \boldsymbol{x}_{t}-\xi_{t}\right)$

(5)

Eq. 5 is differentiated with respect to $w$ and $\xi_{t}$, and, assuming stationary conditions, Eqs. 6 and 7 are obtained.

$\boldsymbol{w}=\frac{1}{2 \lambda} \sum_{t=1}^{T} \alpha_{t} x_{t}$

$\xi_{t}=\frac{\alpha_{t}}{2}$

By substituting the obtained values $w$ and $\xi_{t}$ into Eq. 5, we obtain Eq. 8 .

$$
\begin{array}{r}
W(\boldsymbol{\alpha})=-\frac{1}{4 \lambda} \sum_{s, t=1}^{T} \alpha_{s} \alpha_{t}\left(\boldsymbol{x}_{s} \cdot \boldsymbol{x}_{t}\right)- \\
\frac{1}{4} \sum_{t=1}^{T} \alpha_{t}^{2}+\sum_{t=1}^{T} y_{t} \alpha_{t}(8)
\end{array}
$$

Furthermore, this equation can be re-written in vector form as follows:

$W(\boldsymbol{\alpha})=\boldsymbol{y}^{\prime} \boldsymbol{\alpha}-\frac{1}{4 \lambda} \boldsymbol{\alpha}^{\prime} \boldsymbol{K} \boldsymbol{\alpha}-\frac{1}{4} \boldsymbol{\alpha}^{\prime} \boldsymbol{\alpha}$.

denoting $\boldsymbol{K}$ as the $T \times T$ matrix of dot products. Eq. 9 is differentiated with respect to $\boldsymbol{\alpha}$, and assuming stationary conditions, we obtain the condition 
$-\frac{1}{2 \lambda} \boldsymbol{K} \boldsymbol{\alpha}-\frac{1}{2} \boldsymbol{\alpha}+\boldsymbol{y}=0$.

Eq. 10 is equivalent to

$\boldsymbol{\alpha}=2 \lambda(\boldsymbol{K}+\lambda \boldsymbol{I})^{-1} \boldsymbol{y}$.

Therefore, a prediction $f(\boldsymbol{x})$ of a new unlabeled example $\boldsymbol{x}$

$f(x)=w \cdot x=y^{\prime}(K+\lambda I)^{-1} \boldsymbol{k}$

where $\boldsymbol{k}$ is a vector whose elements are $k_{t}=\boldsymbol{x}_{t} \cdot \boldsymbol{x}$, $t=1, \ldots, T$.

To construct the linear regression in feature space, $\boldsymbol{K}$ can be replaced with the kernel matrix $\boldsymbol{K}_{s, t}=K\left(\boldsymbol{x}_{s}, \boldsymbol{x}_{t}\right)$, and $\boldsymbol{k}$ can be re-written into a vector whose elements are $k_{t}=$ $K\left(\boldsymbol{x}_{t} \cdot \boldsymbol{x}\right)$. In this research, $f(x)$ is the estimated BP value, and $y$ is the measured BP value, with $x_{t}$ representing input variables from the voice spectrum, and $x$ representing the learning variable.

A kernel function, $K\left(\boldsymbol{x}_{s}, \boldsymbol{x}_{t}\right)$, calculates the dot product of the two vectors, $x_{s}, x_{t}$, in a given feature mapping, which are realized in several forms. In this research, a derived Gaussian kernel (Eq. 13) was selected empirically.

$K\left(\boldsymbol{x}_{t}, \boldsymbol{x}\right)=\exp \left(\frac{-a\left\|x_{t}-x\right\|}{\sigma^{2}}\right)$

\section{EVALUATION AND RESULTS}

Initially, one dataset was selected as the evaluation data from the 60 datasets. The remaining 59 were regarded as learning sets, and the BP was then estimated. Next, a different dataset was selected as the new evaluation data, and the BP was estimated from the remaining 59 learning sets. This selection and estimation procedure was repeated 60 times, resulting in 60 sets of BP estimations. The same procedure was performed separately for each subject's dataset.

To provide a performance measurement for the BP estimation, the correlation coefficient between the measured $\mathrm{BP}$ and the estimated BP from spectral components in the voice was used as an evaluation index.

Fig. 6 shows the accuracy performance for systolic BP estimations during SFS selection, by using spectral components from the male subject's voice. Fig. 7 likewise illustrates the accuracy performance for systolic BP estimations for the spectral components from the female subject's voice selected by the SFS method.

Figs. 8 and 9 show the accuracy performances for diastolic BP estimation in the male and female subjects, respectively.

\section{DISCUSSION}

The goal of this research was to estimate BP from the spectral components in voice. As demonstrated in Section 3, diastolic and systolic BP is not non-correlated with specific frequency bands in the subjects' voices. The absolute maximum value of the correlation coefficient exceeded 0.6. These tendencies were witnessed in both the male and female subjects.

However, these correlations were not sufficiently strong to estimate BP with voice-spectrum analysis in a simple way. Hence, the KRR algorithm was used to estimate BP from spectral components in the voice. This KRR-based BP estimation was performed with each subject's data. The results showed that the accuracy performance was saturated when seven or fewer selected explanatory variables were used. The correlation coefficients between both diastolic and systolic BP and the spectral components in the voice exceeded 0.97 in both male and female subjects.

Thus, BP is to some extent correlated with specific spectral components in voice, and the estimated BP applied with KRR was highly correlated to the actual BP. Arterial stiffness varies according to changes in the BP level. Thus, we believe that this influences fluctuations in the vocal tract. As a result, the spectral characteristics of the voice are altered. If this hypothesis is correct, the relationship between BP and voice can be modeled, and a precise estimation can be realized without KRR.

Incidentally, this research is constrained by individual differences in the voice spectrum. It is well known that the spectral characteristics of voice are unique to each person. In this feasibility study, individual differences in the voice spectrum have not been sufficiently examined. In future work, individual differences will be evaluated in a study involving more subjects.

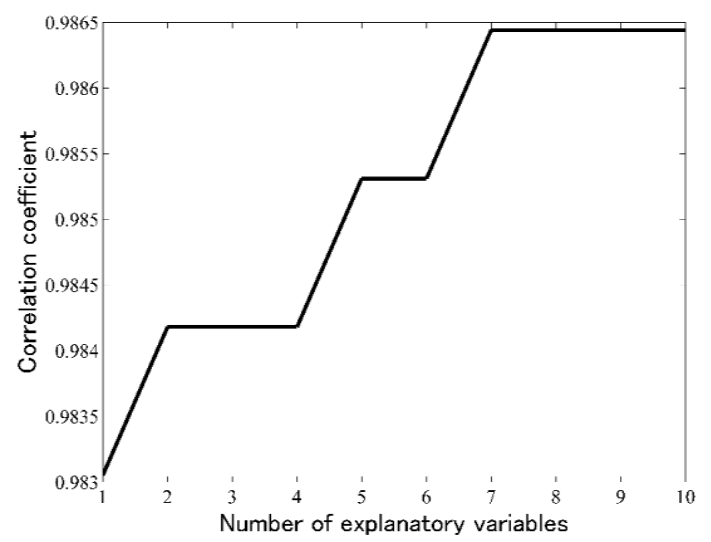

Fig 6: Performance measurement for systolic $B P$ estimations using explanatory variables selected with SFS (male subject)

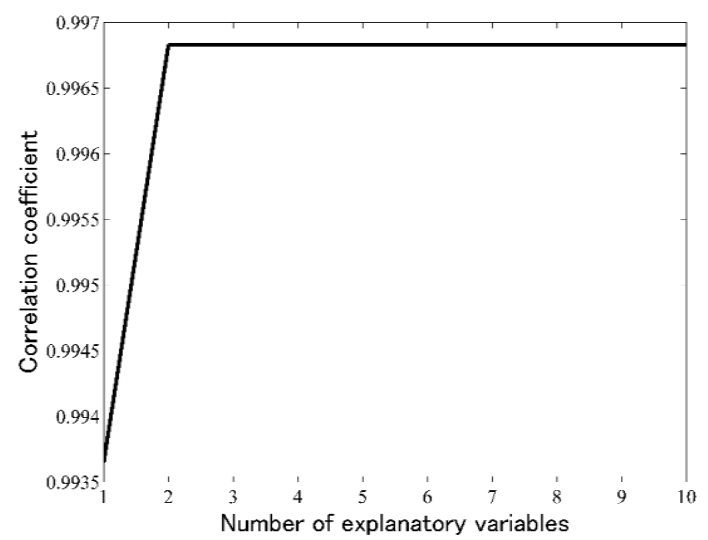

Fig 7: Performance measurement for systolic BP estimations using explanatory variables selected with SFS (female subject)

\section{CONCLUSION}

The purpose of this research was to estimate diastolic and systolic BP with voice-spectrum analysis. First, BP and voice data were measured in male and female subjects, and the correlation coefficients were calculated. This initial analysis 
revealed that $\mathrm{BP}$ and certain components of the voice spectrum are not non-correlated at specific frequency bands. Next, KRR was used as a BP-estimation algorithm applying spectral components of voice. Using this method, BP levels could be estimated with a high degree of accuracy. However, the individual differences in voice have not been adequately reviewed. In future research, individual differences will be investigated in a study involving more subjects. Then, relation between $\mathrm{BP}$ and voice will be modeled.

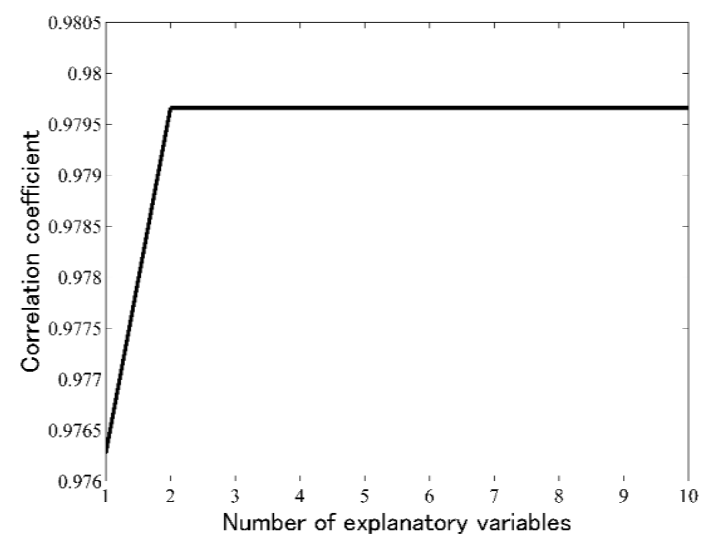

Fig 8: Performance measurement for diastolic BP estimations using explanatory variables selected with SFS (male subject)

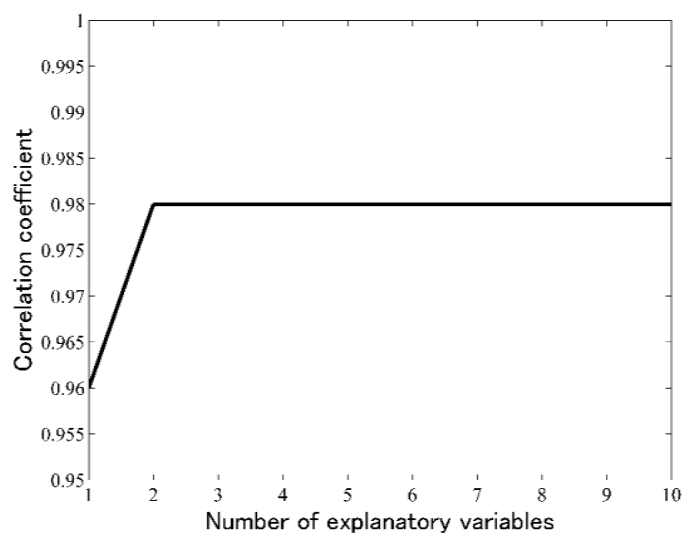

Fig 9: Performance measurement for diastolic BP estimations using explanatory variables selected with SFS (female subject)

\section{REFERENCES}

[1] M. Lida, K. Ueda, A. Okayama, K. Kodama, K. Sawai , S. Shibata, S. Tanaka, T. Keijnkai, H. Horibe, M. Minowa, H. Yanagawa, and T. Hashimoto, "Impact of elevated blood pressure on mortality from all causes, cardiovascular diseases, heart disease and stroke among Japanese: 14 year follow-up of randomly selected population from Japanese - Nippon data 80," J Hum Hypertens, vol. 17, no. 12, pp. 851-857, 2003.

[2] G. Celik, H. Tireli, and H. Narci, "The Impact of Initial Blood Pressure on Early Motality of Patients Suffering an Acute Stroke, " J Neurol Transl Neurosci, vol. 2, no. 1, pp.1031-1035, 2013.

[3] K. Dasgupta, R. R. Quinn, K. B. Zarnke, D. M. Rabi, P. Ravani, S. S. Daskalopoulou, S. W. Rabkin, L. Trudeau, R. D. Feldman, L. Cloutier, A. Prebtani, R. J. Herman, S. L. Bacon, R. E. Gilbert, M. Ruzicka, D. W. McKay, T. S. Campbell, S. Grover, G. Honos, E. L. Schiffrin, P. Bolli, T. W. Wilson, P. Lindsay, M. D. Hill, S. B. Coutts, G. Gubitz, M. Gelfer, M. Vallée, G.V. R. Prasad, M. Lebel, D. McLean, J. M. O. Arnold, G. W. Moe, J. G. Howlett, J. Boulanger, P. Larochelle, L. A. Leiter, C. Jones, R. I. Ogilvie, V. Woo, J. Kaczorowski, K. D. Burns, R. J. Petrella, S. Hiremath, A. Milot, J. A. Stone, D. Drouin, K. L. Lavoie, M. Lamarre-Cliche, G. Tremblay, P. Hamet, G. Fodor, S. G. Carruthers, G. B. Pylypchuk, E. Burgess, R. Lewanczuk, G. K. Dresser,S. B. Penner, R. A. Hegele, P. A. McFarlane, M. Khara, A. Pipe, P. Oh, P. Selby, M. Sharma, D. J. Reid, S. W. Tobe,R. S. Padwal, and L. Poirier, "The 2014 Canadian Hypertension Education Program Recommendations for Blood Pressure Measurement, Diagnosis, Assessment of Risk, Prevention, and Treatment of Hypertension, " Can J Cardiol, vol. 30, no. 5, pp. 485-501, 2014.

[4] Walter N. Kernan, Chair, Bruce Ovbiagele, Henry R. Black, Dawn M. Bravata, Marc I. Chimowitz, Michael D. Ezekowitz, Margaret C. Fang, Marc Fisher, Karen L. Furie, Donald V. Heck, S. Claiborne (Clay) Johnston, Scott E. Kasner, Steven J. Kittner, Pamela H. Mitchell, Michael W. Rich, DeJuran Richardson, Lee H. Schwamm, and John A. Wilson, "Guidelines for the Prevention of Stroke in Patients With Stroke and Transient Ischemic Attack," American Heart Association/American Stroke Association, 2014.

[5] D. Gasperin, G. Netuveli, J. S. Dias-da-Costa, and M. P. Pattussi, "Effect of psychological stress on blood pressure increase: a meta-analysis of cohort studies," Cad. Saúde Pública, vol. 25, no. 4, pp. 715-726, 2009.

[6] K. Chang, D. Fisher, and J. Canny, "AMMON: A Speech Analysis Library for Analyzing Affect, Stress, and Mental Health on Mobile Phones," Proceedings of the 2nd International Workshop on Sensing Applications on Mobile Phones, 2011.

[7] M. O. Mendez, J. Corthout, S. Van Huffel, M. Matteucci, T. Penzel, S. Certti, and A. M. Bianchi, "Automatic screening of obstructive sleep apnea from the ECG based on empirical mode decomposition and wavelet analysis," Physiol Meas, vol. 31 no. 3, pp. 273-289, 2010.

[8] D. Basak, S. Pal, and D. C. Patranabis, "Support Vector Regression," Neural Information Processing, vol. 10, no. 10, pp. 203-224, 2007.

[9] V. N. Vapnik, "Statistical learning theory," In A. Gammerman, editor, Computational Learning and Probabilistic Reasoning. Wiley, 1996.

[10] C. Saunders, A. Gammerman, and V. Vovk, "Ridge Regression Learning Algorithm in Dual Variables," Proceedings of the 15th International Conference on Machine Learning, ICML'98, pp. 515-521, 1998. 